[21] F. H. Chu and K. L. Wong, "Internal coupled-fed dual-loop antenna integrated with a USB connector for WWAN/LTE mobile handset," IEEE Trans. Antennas Propag., vol. 59, no. 11, pp. 4215-4221, Nov. 2011.

[22] K. L. Wong and M. T. Chen, "Small-size LTE/WWAN printed loop antenna with an inductively coupled branch strip for bandwidth enhancement in the tablet computer," IEEE Trans. Antennas Propag., vol. 61, no. 12, pp. 6144-6151, Dec. 2013.

[23] B. Yuan, Y. Cao, and G. Wang, "A miniaturized printed slot antenna for six-band operation of mobile handsets," IEEE Antennas Wireless Propag. Lett., vol. 10, pp. 854-857, Aug. 2011.

[24] B. Yuan, Y. Cao, G. Wang, and B. Cui, "Slot antenna for metalrimmed mobile handsets," IEEE Antennas Wireless Propag. Lett., vol. 11, pp. 1334-1337, Nov. 2012

[25] J. Zhong, K. K. Chen, and X. Sun, "A novel multi-band antenna for mobile phone with metal frame," in Proc. Int. Conf. Wireless Commun. Netw. Mobile Comput., Shanghai, China, 2012, pp. 1-4.

[26] J. Lee, K. Min, and Y. Cheon, "Miniaturized antennas with reduced hand effects in mobile phones using magneto-dielectric material," IEEE Antennas Wireless Propag. Lett., vol. 13, pp. 935-938, May 2014.

[27] Z. N. Chen, N. Yang, Y. X. Guo, and Y. W. Chia, "An investigation into measurement of handset antennas," IEEE Trans. Instrum. Meas., vol. 54, no. 3, pp. 1100-1110, Jun. 2005.

[28] N. Behdad and K. Sarabandi, "Dual-band reconfigurable antenna with a very wide tenability range," IEEE Trans. Antennas Propag., vol. 54, no. 2, pp. 409-416, Feb. 2006

[29] D. Peroulis, K. Sarabandi, and L. P. B. Katehi, "Design of reconfigurable slot antenna," IEEE Trans. Antennas Propag., vol. 53, no. 2, pp. 645-654, Feb. 2005.

[30] Index SAR [Online]. Available: http://www.indexsar.com

\section{Modeling of Nonresonant Longitudinal and Inclined Slots for Resonance Tuning in ENZ Waveguide Structures}

Nebojsa Vojnovic, Branka Jokanovic, Milos Radovanovic, Francisco Medina, and Francisco Mesa

\begin{abstract}
Novel methods are presented for the independent resonance tuning in rectangular waveguide epsilon-near-zero (ENZ) structures. This kind of tuning is achieved using short longitudinal and inclined slots placed in the middle of the ENZ channel. It is demonstrated that the tunneling frequency and Fabry-Pérot resonance (FPR) can be shifted by as much as $8 \%$ and $9.7 \%$, respectively. Equivalent circuits for the tuning slots are given, and their equivalent parameters are extracted and validated. Analytical expressions for the equivalent slot reactances are also provided, and they show very good agreement with the extracted parameters. Experimental verification of both presented tuning methods is provided, and the agreement between simulated and experimental results is excellent (error less than $1 \%$ )
\end{abstract}

Index Terms-Epsilon-near-zero (ENZ) waveguide, equivalent circuit, frequency tuning, waveguide slots.

\section{INTRODUCTION}

A key feature that has made metamaterials so appealing in the past few years is the possibility of tailoring their effective constitutive parameters $\varepsilon$ and $\mu$ in such a way as to suit the specific needs of the application at hand. A subclass of metamaterials that has attracted a significant interest is the so-called epsilon-near-zero (ENZ) media [1]. The dispersion characteristics of a rectangular waveguide operating near the fundamental mode cutoff frequency were first used in [2] as a possibility of realizing ENZ metamaterials. Other types of waveguides such as SIW [3] and double-ridge [4] can also be employed as a basis to implement artificial ENZ metamaterials. Considering the features of these media, potential applications in dielectric sensing [3], [5], miniaturization [6], "supercoupling," and lens synthesis [7], [8] are actually expected.

One of the most interesting and exploited property of ENZ waveguide structures is their inherent ability to "squeeze" and tunnel electromagnetic (EM) energy through very tight waveguide channels. Theoretical discussion about these seemingly anomalous phenomena can be found in [1], and experimental verification is given, for instance, in [9]. Since the tunneling condition is met only at one specific frequency, it would be very convenient to have some control on the tunneling frequency in order to meet different specifications. Thus,

Manuscript received December 28, 2014; revised July 13, 2015; accepted August 18, 2015. Date of publication August 26, 2015; date of current version October 28, 2015. This work was supported by the Serbian Ministry of Education, Science and Technological Development through the Project TR32024 and in part by the Project of Bilateral Cooperation between the Kingdom of Spain and the Republic of Serbia PRI-AIBSE-2011-1119. The work of F. Mesa and F. Medina was supported in part by the Spanish Ministry of Economy and Competitiveness under Contract TEC2013-41913-P and in part by the Spanish Junta de Andalucía under Contract P12-TIC-1435.

N. Vojnovic, B. Jokanovic, and M. Radovanovic are with the Institute of Physics, University of Belgrade, 11080 Belgrade, Serbia (e-mail: nebojsav@ipb.ac.rs; brankaj@ipb.ac.rs; rmilos@ipb.ac.rs).

F. Medina is with the Department of Electronics and Electromagnetism, Faculty of Physics, University of Seville, 41012 Seville, Spain (e-mail: medina@us.es).

F. Mesa is with the Department of Applied Physics, University of Seville, 41012 Seville, Spain (e-mail: mesa@us.es).

Color versions of one or more of the figures in this communication are available online at http://ieeexplore.ieee.org.

Digital Object Identifier 10.1109/TAP.2015.2473700 
in [10], the tunneling frequency of the ENZ waveguide structure was altered using a varactor diode in the middle of the channel accompanied by an appropriate biasing network. However, this method is not easy to apply to the ENZ structures with thinner channels. In a recent paper published by some of the authors [11], two longitudinal nonresonant slots were placed at the channel ends as elements for simultaneous tuning of "zero-order resonance" (ZOR) and FabryPérot resonances (FPR) of the considered structure. Theoretical results were also confirmed experimentally. In the present communication, we describe and experimentally verify methods for the independent tuning of the two kinds of resonances in the ENZ channel. The availability of a method to tune each of those resonances separately has obvious advantages. This goal is achieved in this communication by the proper use and disposition of short slots. Equivalent circuits for ENZ waveguides with tuning slots are also presented as well as the extracted slot parameters and their analytical expressions as functions of the operating frequency. It should be noticed that the slots under consideration are electrically short and, to the authors' knowledge, analytical expressions describing their impedances have not been reported in the literature so far.

\section{ENZ WAVEGUIDE-INitial Structure}

The basic ENZ waveguide system considered in this communication consists of two input/output waveguides connected by means of a thin channel [see Fig. 1(a) for relevant dimensions; metallization and segments filled with different dielectrics are clearly visible]. Waveguide ports are placed on the leftmost and rightmost sides of the structure.

\section{A. Equivalent Circuit Extraction Procedure}

The equivalent circuit of this structure is depicted in Fig. 1(b). $Z_{\mathrm{w}}^{c}$ and $Z_{\mathrm{ch}}^{c}$ denote characteristic impedances of the input/output waveguides and channel, respectively, while $\gamma_{\mathrm{w}}$ and $\gamma_{\mathrm{ch}}$ denote the propagation constants of the fundamental modes in those waveguide sections. The abrupt $E$-plane steps between the input/output waveguides and the channel are accounted for by the capacitors with capacitance $C$ [12]. The characteristic impedances of the equivalent transmission lines are calculated as

$$
Z_{\mathrm{w}(\mathrm{ch})}^{c}=\frac{2 b_{\mathrm{w}(\mathrm{ch})}}{a} Z_{\mathrm{w}(\mathrm{ch})}^{w}=\frac{\left(2 b_{\mathrm{w}(\mathrm{ch})} / a\right) \omega \mu}{\sqrt{\omega^{2} \mu_{0} \varepsilon_{0} \varepsilon_{\mathrm{r}, \mathrm{w}(\mathrm{ch})}-(\pi / a)^{2}}}
$$

where $Z_{\mathrm{w}(\mathrm{ch})}^{w}$ stand for wave impedances of the $\mathrm{TE}_{10}$ modes (input/output waveguides or channel). In this work, we use an extraction method to obtain $C$ based on the comparison of the response of the equivalent circuit in Fig. 1(b) (whose closed-form expression can readily be obtained) with the full-wave data computed with the commercial software Ansys HFSS [13]. Only the $S_{11}$-parameter is required in this extraction as $C$ is the only unknown in our circuit model. Results from HFSS are obtained using adaptive mesh refinement with a convergence criterion defined so that the difference between the magnitudes of $S$-parameters calculated in successive iterations is less than $0.1 \%$. First-order basis functions are taken over the tetrahedral mesh elements. In order to avoid any discrepancies due to solver interpolation, discrete sweeps with a $10 \mathrm{MHz}$ resolution were employed. The extracted values for $C$ are very close to the ones calculated with the formulas in [12] provided the operation frequency is not too close to the cutoff frequency of the fundamental mode in the input/output sections.

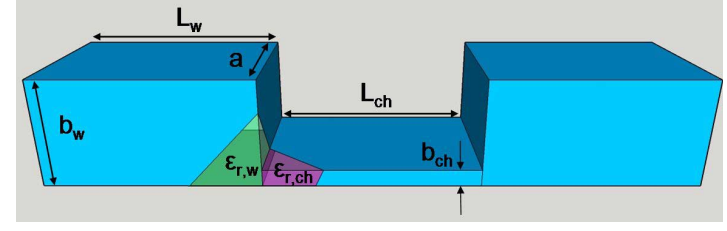

(a)

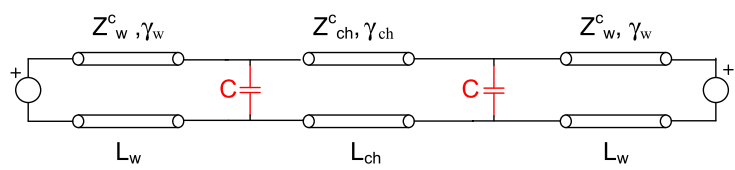

(b)

Fig. 1. (a) Initial ENZ waveguide structure; widths and heights of input/output waveguides are $a=23 \mathrm{~mm}$ and $b_{\mathrm{w}}=10 \mathrm{~mm}$, respectively; the height of the channel is $b_{\mathrm{ch}}=0.7 \mathrm{~mm}$ and the lengths of both the input waveguides and the channel section are $L_{\mathrm{w}}=L_{\mathrm{ch}}=32 \mathrm{~mm}$. (b) Equivalent circuit for the structure in (a). Dielectric materials: $\varepsilon_{\mathrm{r}, \mathrm{w}}=2.1, \tan \delta=0.0009$ (Teflon), and $\varepsilon_{\mathrm{r}, \mathrm{ch}}=1$ (air).

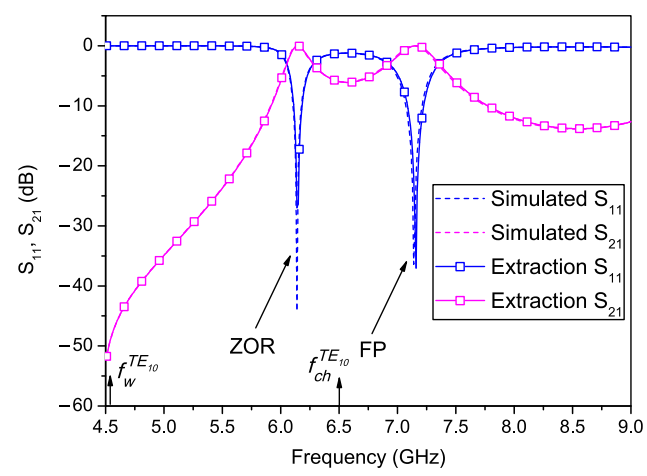

Fig. 2. Simulated (HFSS) and analytically computed (circuit model) $S$-parameters for the structure in Fig. 1(a).

\section{B. Resonances in ENZ Waveguide}

Simulated $S$-parameters of the structure from Fig. 1(a) and $S$-parameters calculated using the extracted values of the shunt capacitances are compared in Fig. 2. Good agreement is observed over the whole frequency band. The two observed resonances are well below the cutoff frequency of the $\mathrm{TE}_{20}$ mode in the channel and input/output waveguides, thus single mode operation $\left(\mathrm{TE}_{10}\right)$ is ensured. As already described in [1], these two resonances are very different in nature. The first resonance at $6.14 \mathrm{GHz}$ is, in fact, a consequence of the ENZ tunneling effect (ZOR) while the second one at $7.15 \mathrm{GHz}$ is a regular FPR. It should be noted that the position of ZOR can be affected by changing the channel length. This phenomenon is due to the fact that the stored magnetic energy in the channel region increases with the channel length while the shunt capacitance remains the same. Fig. 3(a) and (b) shows the surface current density vector on the top of the channel at these two resonances. It has been checked that the electric field distribution shows no phase delay at ZOR (tunneling effect) while at FPR it shows a clear standing wave pattern.

As reported in [14], the equivalent circuit in Fig. 1(b) can be analytically solved to obtain both the ZOR and FPR conditions. These conditions can be expressed as transcendental quadratic equations derived from the appropriate condition on the input impedance of the channel, which can be graphically or numerically solved. 


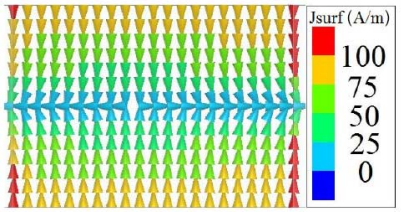

(a)

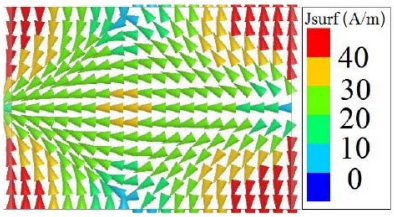

(b)
Fig. 3. Surface current density vector along the channel at (a) ZOR and (b) FPR.

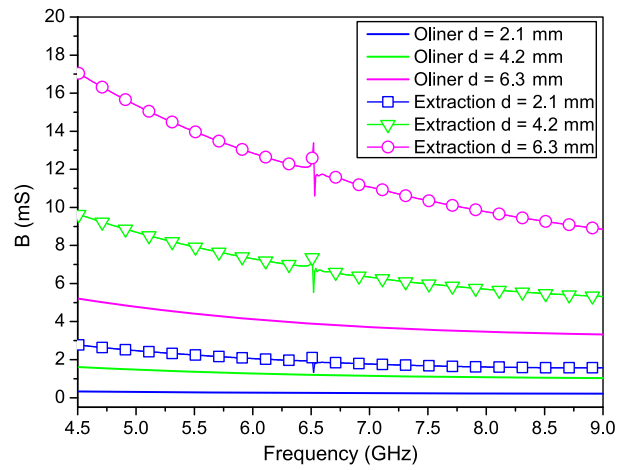

Fig. 4. Extracted versus analytically calculated [15, eq. (12)] susceptances of longitudinal offset $(d)$ slot placed on the top wall of a narrow $\left(b_{\mathrm{ch}}=0.7 \mathrm{~mm}\right)$ hollow waveguide. Width of the waveguide is $a=23 \mathrm{~mm}$, length and width of the slot are $a_{\mathrm{s}}=10 \mathrm{~mm}$ and $b_{\mathrm{s}}=0.4 \mathrm{~mm}$, respectively.

\section{Treatment of Short Longitudinal Slots}

As mentioned above, electrically short slots made on the wide side of the channel section will be employed as tuning elements. Slots in standard waveguides (such as the WR90) can be modeled by using the formulas provided in [15]. Alternatively, the real and imaginary parts of the slot admittances can be obtained using an extraction procedure similar to the one used to calculate $C$ in Fig. 1(b). For standard waveguides and resonant slots, the extraction method yields results in good agreement with [15, eqs. (11) and (12)]. However, in Fig. 4, it is shown that the extracted and analytically calculated susceptances do not agree for narrow slots in waveguides having reduced height (channel). Hence, the numerical extraction procedure will be employed in this work to characterize the tuning slots.

\section{ENZ Channel With a Centered Longitudinal Slot}

In [11], it was demonstrated and experimentally verified that placing two identical offset longitudinal slots at the input and output ends of the channel results in a frequency shift of both ZOR and FPR. In this section, it will be explored the effect that placing a single longitudinal offset slot in the middle of the channel [see Fig. 5(a)] has on the resonances of the structure. Fig. 5(b) shows the equivalent circuit for the structure in Fig. 5(a). As the short longitudinal slot intercepts transverse currents, it will be modeled as a shunt lumped capacitor (radiation is assumed negligible, and radiation resistance is then ignored). The influence of a single offset slot on both resonances is plotted in Fig. 6, which shows both numerical and experimental results. This figure clearly shows that the longitudinal offset slot only modifies the ZOR frequency with meaningless influence on the FPR. As the slot offset is increased, the value of the ZOR frequency is reduced. The largest frequency shift is observed for a slot offset of $8.4 \mathrm{~mm}$ and it is equal to $4 \%$ when compared to the case with no slots. Maximum

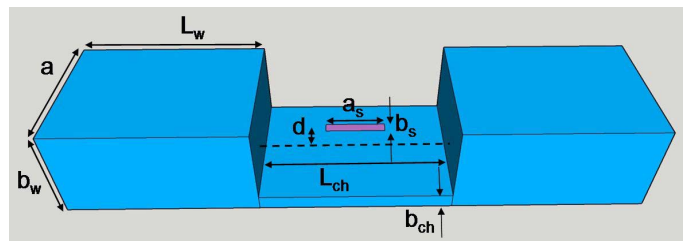

(a)

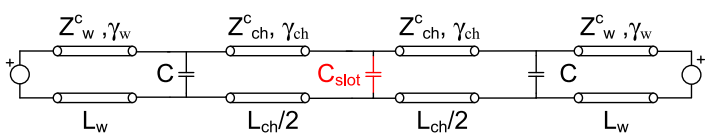

(b)

Fig. 5. (a) ENZ channel with a single offset (d) longitudinal slot in the middle of the channel. (b) Equivalent circuit for the structure in (a).

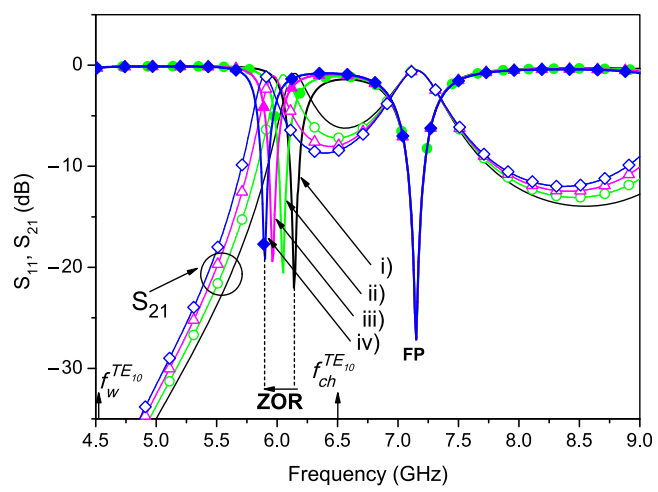

(a)

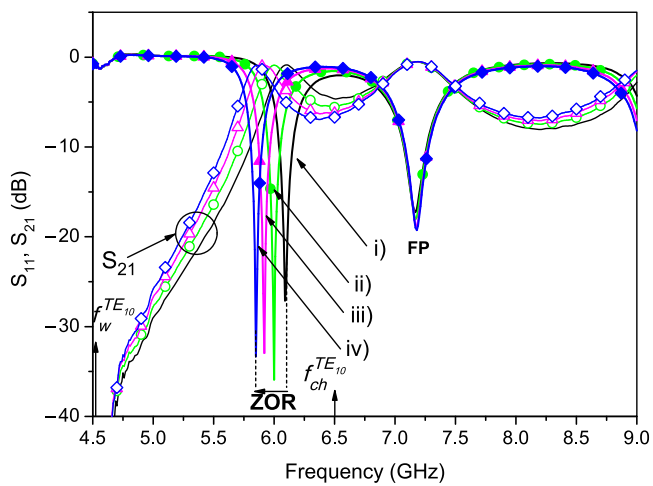

(b)

Fig. 6. Influence of a single-centered offset slot on ZOR and FPR ( $a_{s}=10 \mathrm{~mm}, b_{s}=0.4 \mathrm{~mm}$ ). (i) $S_{11}$, no slot. (ii) $S_{11}, d=4.2 \mathrm{~mm}$. (iii) $S_{11}$, $d=6.3 \mathrm{~mm}$. (iv) $S_{11}, d=8.4 \mathrm{~mm}$. $S_{21}$ curves are plotted with dotted lines and various symbols. (a) Simulation. (b) Measurement.

slot length was chosen so as to get the largest ZOR tuning range without disturbing the position of the FPR. If the materials used to build the ENZ structure are assumed lossless, the magnitude

$$
\rho=1-\left|S_{11}\right|^{2}-\left|S_{21}\right|^{2}
$$

is a measure of the relative power radiated through the slot. This radiated power is of the order of $\sim 10^{-2}$, being greater for larger offsets. Moreover, the radiation losses are pronounced only at ZOR but not at FPR. 


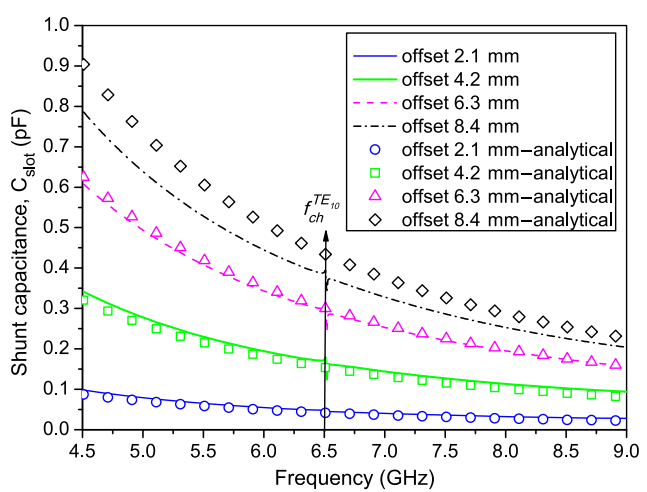

Fig. 7. Frequency dependence of the extracted slot shunt capacitance for four different slot offsets. Extracted values are compared with those obtained analytically using (5).

\section{A. Analytical Expressions for the Slot Capacitance}

Since the extraction method employed in this work for obtaining the slot capacitance inevitably requires a very time-consuming 3-D simulation in the whole frequency range of interest, it would be very convenient to have analytical expression for those capacitances based on a single frequency point simulation. Such possibility will be demonstrated for two situations in this section. In the first case, $b_{\mathrm{ch}}$ is kept fixed while the slot offset is varied. The second case implies an extension of the first case in the sense that a variable channel height is introduced.

In order to derive an expression for $C_{\text {slot }}$, we make use of [15, eqs. (11) and (12)], which read in our notation as

$$
\frac{X}{Z_{\mathrm{ch}}^{c}}=\frac{B_{c}}{Y_{\mathrm{ch}}^{c}} V_{\mathrm{sh}}^{2}
$$

where the transformation number $V_{\mathrm{sh}}$ is given by

$$
V_{\mathrm{sh}}=\left(\frac{2 a}{\lambda_{g}}\right)\left[\frac{\cos \left(\frac{\pi a_{s}}{2 a}\right)\left[1-\left(\frac{2 a_{s}}{\lambda_{g}}\right)^{2}\right]}{\cos \left(\frac{\pi a_{s}}{\lambda_{g}}\right)\left[1-\left(\frac{a_{s}}{a}\right)^{2}\right]}\right] \csc \left(\frac{\pi d}{a}\right)
$$

where $X$ is the reactance of the slot in question and $B_{c}$ is the susceptance of a longitudinal slot with no offset $(d=0)$. As it was discussed in Fig. 4, the susceptances calculated in this manner do not agree well with the extracted ones for thin channels. However, since the dependence on $d$ in (4) only appears in the cosecant factor, the following approximate expression for $C_{\text {slot }}$ is proposed:

$$
C_{\text {slot }}=C_{0}^{b}\left(\frac{f_{0}}{f}\right)^{2} \frac{\sin ^{2}\left(\frac{\pi d}{a}\right)}{\sin ^{2}\left(\frac{\pi d_{0}}{a}\right)}
$$

where $C_{0}^{b}$ is the numerically extracted value of $C_{\text {slot }}$ at frequency $f_{0}$, for an offset $d=d_{0}$ and a specific value of $b_{\mathrm{ch}}=b$. Frequency $f_{0}$ has been chosen to be equal to the ZOR tunneling frequency and $d_{0}$ equals half of the maximum slot offset (in the present case, $d_{0}=5.75 \mathrm{~mm}$ ). In Fig. 7, the plots corresponding to the values of capacitances calculated using (5) (denoted as "analytical") are compared with the ones obtained from the numerical extraction method. The observed agreement is good except for the case $d=8.4 \mathrm{~mm}$, as a consequence of the increasing coupling between the slot and the waveguide sidewall.

If different channel heights have to be explored, the following expression is proposed for $C_{0}^{b}$ in (5):

$$
C_{0}^{b}=2 C_{0}^{b_{0}} \frac{b_{0}}{b} \frac{1}{1+\frac{b}{b_{0}}}
$$

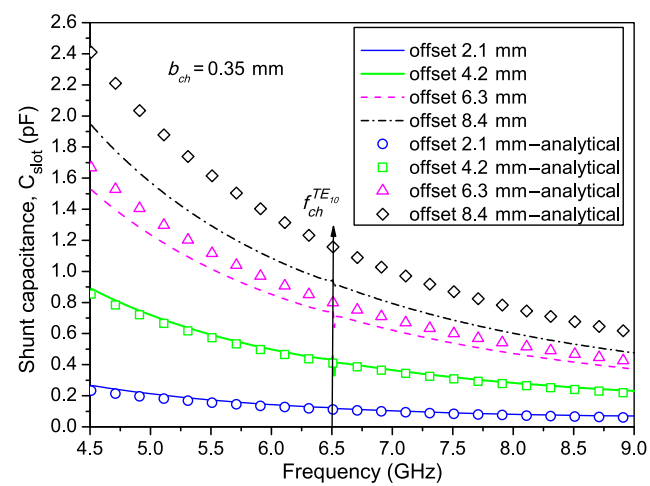

Fig. 8. Frequency dependence of the extracted slot shunt capacitance for four different slot offsets in a thin ENZ channel $\left(b_{\mathrm{ch}}=0.35 \mathrm{~mm}\right)$. Extracted values are compared with those obtained following expression (5).

where $C_{0}^{b_{0}}$ is the slot capacitance numerically extracted for the channel height $b_{\mathrm{ch}}=b_{0}$ at $f=f_{0}$ and $d=d_{0}$. For convenience, the parameter $b_{0}$ is chosen to be equal to the width of the slot. The derivation of relation (6) comes from combining two asymptotic cases of channel height. The first case considers very small $b_{\mathrm{ch}}$. In such case, the capacitances between the waveguide top and bottom horizontal plates are dominant and hence, bearing in mind the definition for the capacitance of a parallel-plate capacitor, the following ratio is found:

$$
C_{0}^{b}=C_{0}^{b_{0}} \frac{b_{0}}{b} .
$$

If $b_{\mathrm{ch}}$ is large, the capacitance between the conductors of only the top plane is dominant, giving

$$
C_{0}^{b}=C_{0}^{b_{0}}\left(\frac{b_{0}}{b}\right)^{2}
$$

Expression (6) estimates the value of $C_{0}^{b}$ at the intersection point of the curves defined by (7) and (8).

After obtaining $C_{\text {slot }}$, the whole equivalent circuit shown in Fig. 5(b) can be solved and its results compared versus those obtained using a full-wave simulation. This comparison (omitted here) shows a very good agreement between the simulated and numerically obtained $S$-parameters with a slight frequency shift below $0.3 \%$. Thus, the assumption about neglecting the real part of the equivalent shunt element in Fig. 5(b) is found to be valid. As observed in Fig. 7, all the extracted capacitances decay with frequency and larger offsets yield greater capacitances. The discontinuity at $6.5 \mathrm{GHz}$ is due to the fundamental mode cutoff in the channel. It is interesting to mention that the extracted single slot capacitances following the procedure in [11] and those from Fig. 7 are almost identical. In other words, the slot capacitance does not seem to depend on its position along the channel (only slot length and offset are relevant).

Fig. 8 shows the extracted $C_{\text {slot }}$ values versus frequency for four different offsets in a thin ENZ channel $\left(b_{\mathrm{ch}}=0.35 \mathrm{~mm}\right)$. These values are compared with those obtained using the analytical expression in (5). The agreement is good with a relatively larger error for the greatest offset $(d=8.4 \mathrm{~mm})$. Same comments as those regarding Fig. 7 apply here. Fig. 9 shows the case of a thicker ENZ channel $\left(b_{\mathrm{ch}}=2 \mathrm{~mm}\right)$. In this case, the agreement is very good for all the four offsets.

It is interesting to note that the tuning range can be easily increased using multiple slots. For instance, placing two longitudinal slots along the channel, one in the channel top conducting plane and the other one in the bottom plane (in order to minimize the coupling between the slots) with the same longitudinal coordinate, the tuning range can 


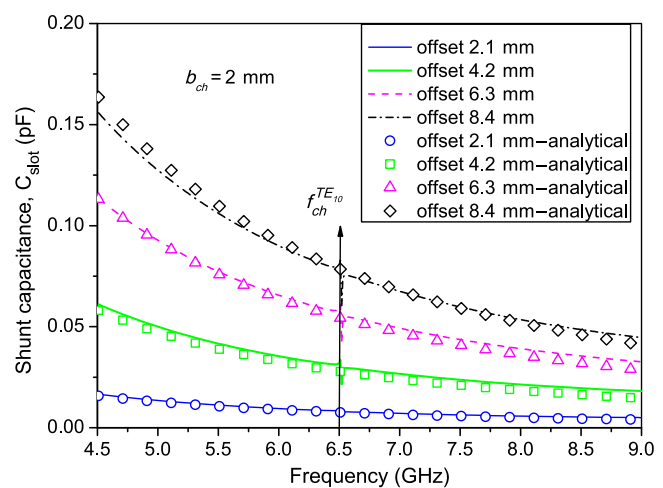

Fig. 9. Frequency dependence of the extracted slot shunt capacitance for four different slot offsets in a thick ENZ channel $\left(b_{\mathrm{ch}}=2 \mathrm{~mm}\right)$. Extracted values are compared with those obtained following expression (5).

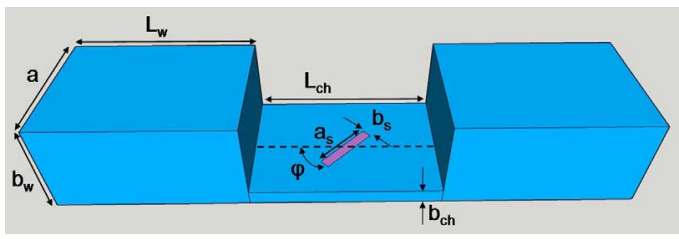

(a)

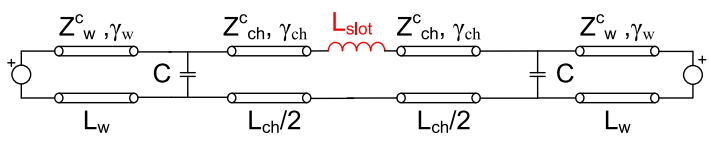

(b)

Fig. 10. (a) ENZ channel with a centered inclined slot cut in the middle of the channel top side. (b) Equivalent circuit for the structure in (a).

be doubled to $8 \%$ for the largest considered offset. Thus, from the previous discussion and the results in [11], it can be concluded that two longitudinal slots can be placed along the channel in different configurations in order to manipulate the structure resonances in different manners. If the slots are placed directly on the $E$-plane steps, both resonances (ZOR and FPR) are affected. If the two slots are placed in the channel central region, only ZOR can be tuned. Independent ZOR tuning is a direct consequence of positioning longitudinal slots in the middle of the channel. Off-center positioning affects both resonances.

\section{ENZ Channel With a Centered InClined Slot}

It has already been shown that the offset longitudinal slot makes it possible to tune the ZOR frequency without affecting the FPR frequency. In a similar way, it would be very convenient to have a procedure to manipulate the FPR frequency without influencing the ZOR, thus completing the independent resonance tuning mechanism. In order to explore such a possibility, the ENZ structure is now studied with a single-centered slot with no offset but instead with an angle of inclination $\varphi$ relative to the channel longitudinal axis [see Fig. 10(a)]. The equivalent circuit that corresponds to the structure in Fig. 10(a) is given in Fig. 10(b). From the surface currents in Fig. 3(a) and (b), it can be concluded that a centered inclined slot will disturb the axial component of the surface current and, hence, an inductive series element representation is chosen for this case, ignoring once again the radiation losses.

In Fig. 11, the extracted values of the series inductances are given for four different angles of inclination. As this angle increases, the

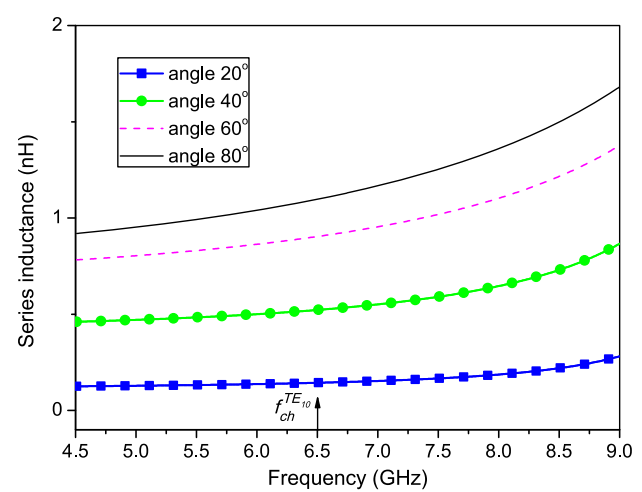

Fig. 11. Frequency dependence of the extracted slot series inductance for four different angles of inclination of the centered slot.

series inductance also increases. A slight growth of these inductances with frequency is also observed, which is more pronounced for larger angles. After comparing the $S$-parameters (omitted here) for the complete structure in Fig. 10(a) obtained using HFSS and those calculated with the equivalent circuit, a good agreement was observed (with a slight frequency shift of less than $0.4 \%$ ). This again justifies the neglecting of the real part of the series element in Fig. 10(b).

The influence of the angle of inclination of the centered slot on ZOR and FPR is shown in Fig. 12(a). This figure shows that a singlecentered inclined slot causes a frequency shift of the FPR only. As the angle of inclination is increased, the FPR moves to lower frequencies while the ZOR remains at the same position. The largest frequency shift is observed for a slot angle $\varphi=80^{\circ}$ and is equal to $6.7 \%$ when compared to the case without the slot. Increasing the angle beyond $90^{\circ}$ has no significance due to symmetry. Maximum slot length was chosen in order to obtain the maximum FP tuning range while keeping the position of the ZOR fixed. The radiation losses for this type of slot are appreciable only at FPR but not at the ZOR tunneling frequency. Greater angles of slot inclination give rise to higher radiation losses. The radiation losses are about five times higher in this case than in the previous one.

Similarly to the discussion at the end of Section III, multiple inclined slot configuration could be used in order to additionally widen the FP tuning range. If one slot (with the same dimensions as in the previous example) is placed in the channel top wall and the other in the channel bottom wall (exactly beneath the first one), having the opposite angles of inclination $\varphi$ with respect to the channel center line, the FPR tuning range can be improved up to $9.7 \%$, which represents nearly a $50 \%$ improvement over the single slot case.

\section{Measurement Results}

In order to verify the proposed independent tuning mechanisms in ENZ waveguide, a series of measurements were performed. As seen in Fig. 13, the measurement setup consists of three waveguide sections with upper (slotted) metallic wall removed, which form the considered ENZ structure assembled in a single aluminum block. Input waveguides are filled with Teflon, while the channel is left hollow. Afterward, a copper foil, with appropriate slot geometries obtained using photo-lithography procedure, is placed to enclose the structure. Good electrical contact between the foil and the aluminum block is achieved by means of vacuum tightening as well as an screwedon metallic lid placed over the foil. Input waveguides are excited using coaxial pins, and the obtained results are later de-embedded in order to be directly compared to the wave-port excitation used in fullwave simulations. Measurements were performed using Anritsu VNA 


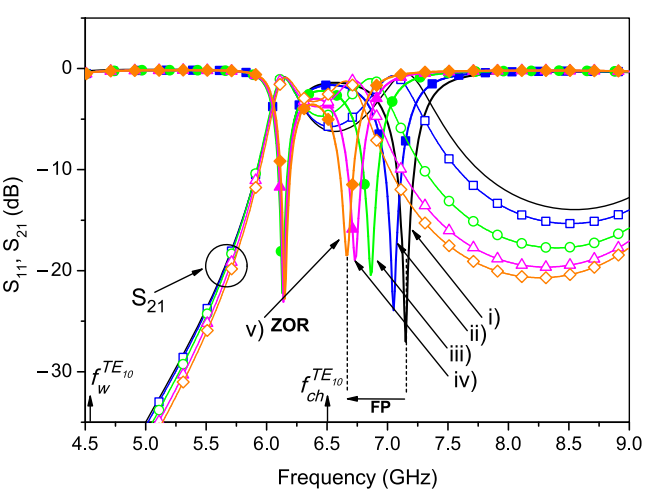

(a)

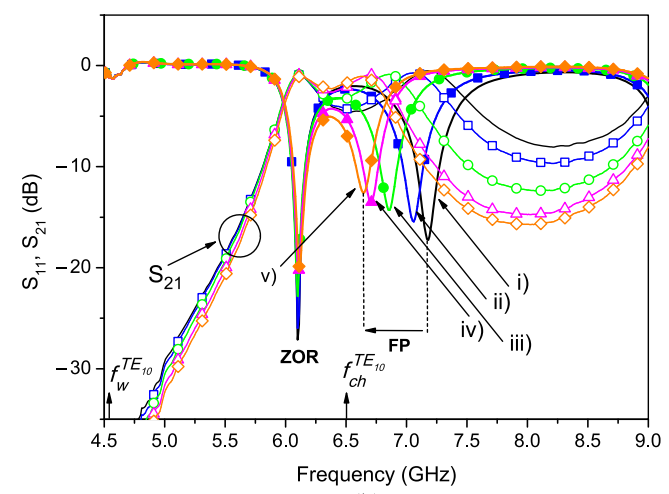

(b)

Fig. 12. Influence of the angle of inclination of a single-centered slot on the ZOR and the FPR $\left(a_{s}=14 \mathrm{~mm}, b_{s}=0.4 \mathrm{~mm}\right)$. (i) $S_{11}$, no slot. (ii) $S_{11}$, $\varphi=20^{\circ}$. (iii) $S_{11}, \varphi=40^{\circ}$. (iv) $S_{11}, \varphi=60^{\circ}$. (v) $S_{11}, \varphi=80^{\circ} . S_{21}$ curves are plotted using dotted lines with appropriate symbols. (a) Simulation. (b) Measurement.

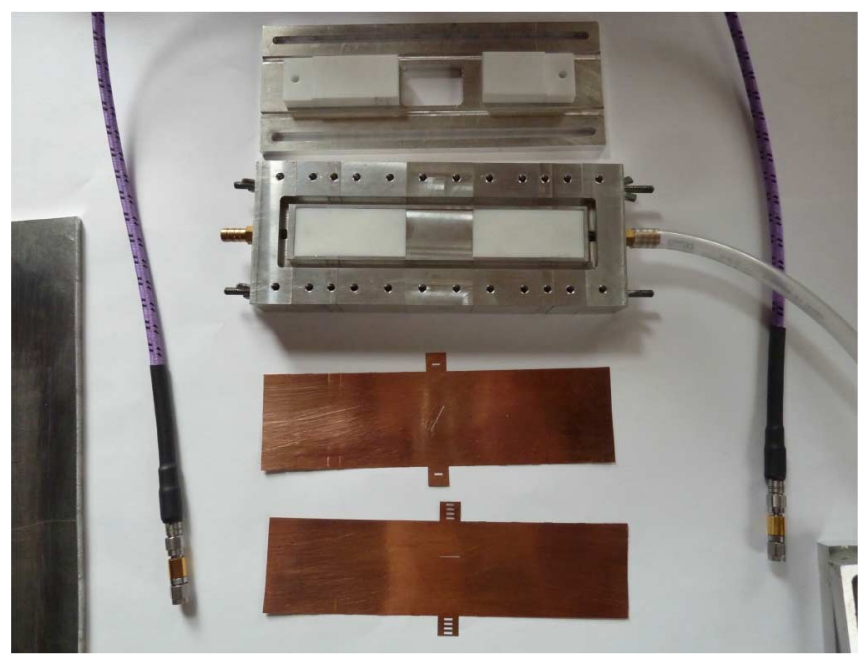

Fig. 13. Disassembled measurement setup consisting of a carved aluminum block and copper foils. Vacuum pump connections, triple offset calibration kit, and metallic lid are also shown.

ME7838 A network analyzer, which was calibrated using a triple offset technique.

Measurement results are shown in Figs. 6(b) and 12(b) for the longitudinal offset and inclined slot geometries, respectively. The expected independent resonance tuning mechanism discussed in this work is clearly validated by these experiments. Compared to the simulated results, an excellent agreement of the resonant frequencies can be observed, with a maximum difference of $50 \mathrm{MHz}$ (error of less than 1\%). Maximum measured insertion loss is better than $1 \mathrm{~dB}$, which agrees well with the simulations which take into account the finite conductivity of both aluminum (38 MS/m) and copper (58 MS/m).

\section{CONCLUSION}

In this communication, we present novel methods for the separate tuning of the ENZ waveguide resonant frequencies. Interesting results are obtained if a single slot is placed in the middle of the channel. Namely, by keeping the slot in the longitudinal form, one can manipulate the position of the tunneling frequency merely by changing the slot offset. In addition, if the slot is rotated with respect to the channel center line, only the position of the FPR is changed. It is shown that the longitudinal offset slot can be modeled by means of an equivalent shunt capacitor in its single and dual configuration, with the same capacitance regardless of its position along the channel (provided its length and offset are the same). On the other hand, rotated slot can be modeled by means of an equivalent series inductor. Based on the extracted equivalent capacitance of the tuning slots, accurate expressions are proposed for the frequency-dependent slot capacitance for different slot offsets and different heights of the ENZ channel. The validity of all the mentioned equivalent circuits has been checked and errors smaller than $0.4 \%$ have been obtained with respect to full-wave simulations. In addition, an experimental verification for the independent resonance tuning mechanisms is demonstrated. The agreement between the measured and simulated results is excellent with an error of less than $1 \%$.

\section{REFERENCES}

[1] M. Silveirinha and N. Engheta, "Tunneling of electromagnetic energy through subwavelength channels and bends using $\varepsilon$-near-zero materials," Phys. Rev. Lett., vol. 97, p. 157403, 2006.

[2] W. Rotman, "Plasma simulation by artificial dielectrics and parallel-plate media," IRE Trans. Antennas Propag., vol. 10, no. 1, pp. 82-85, Jan. 1962.

[3] H. Lobato-Morales, D. V. B. Murthy, A. Corona-Chávez, J. L. OlveraCervantes, J. Martínez-Brito, and L. G. Guerrero-Ojeda, "Permittivity measurements at microwave frequencies using epsilon-near-zero (ENZ) tunnel structure," IEEE Trans. Microw. Theory Techn., vol. 59, no. 7, pp. 1863-1868, Jul. 2011.

[4] B. M. Kim, H. W. Son, Y. K. Cho, and J. P. Hong, "Transmission-line analysis of an epsilon near zero tunneling circuit using a double ridge rectangular waveguide," J. Korean Phys. Soc., vol. 65, no. 5, pp. 625-630, Sep. 2014.

[5] A. Alù and N. Engheta, "Dielectric sensing in $\varepsilon$-near-zero narrow waveguide channels," Phys. Rev. B, vol. 78, p. 045102, Jul. 2008.

[6] J. G. Pollock and A. K. Iyer, "Miniaturized circular-waveguide probe antennas using metamaterial liners," IEEE Trans. Antennas Propag., vol. 63, no. 1, pp. 428-433, Jan. 2015.

[7] V. P. Peña et al., "Mechanical $144 \mathrm{GHz}$ beam steering with all-metallic epsilon-near-zero lens antenna," Appl. Phys. Lett., vol. 105, p. 243503 , 2014

[8] V. Torres et al., "Experimental demonstration of a millimeter-wave metallic ENZ lens based on the energy squeezing principle," IEEE Trans. Antennas Propag., vol. 63, no. 1, pp. 231-239, Jan. 2015.

[9] B. Edwards, A. Alù, M. E. Young, M. Silveirinha, and N. Engheta, "Experimental verification of epsilon-near-zero metamaterial coupling and energy squeezing using a microwave waveguide," Phys. Rev. Lett., vol. 100, p. 033903, Jan. 2008.

[10] D. A. Powell, A. Alù, B. Edwards, A. Vakil, Y. S. Kivshar, and N. Engheta, "Nonlinear control of tunneling through an epsilon-near-zero channel," Phys. Rev. B, vol. 79, p. 245135, Jun. 2009.

[11] M. Mitrovic, B. Jokanovic, and N. Vojnovic, "Wideband tuning of the tunneling frequency in a narrowed epsilon-near-zero channel," IEEE Antennas Wireless Propag. Lett., vol. 12, pp. 631-634, May 2013.

[12] N. Marcuvitz, Waveguide Handbook. New York, NY, USA: McGrawHill, 1951.

[13] [Online]. Available: http://www.ansys.com 
[14] N. Vojnovic, M. Mitrovic, and B. Jokanovic, "Modeling of tunneling effects in epsilon-near-zero waveguide," Microw. Rev., vol. 18, pp 21-27, 2012.

[15] A. Oliner, "The impedance properties of narrow radiating slots in the broad face of rectangular waveguide: Part II-Comparison with measurement," IRE Trans. Antennas Propag., vol. 5, no. 1, pp. 12-20, Jan. 1957.

\section{A Simple Model for Average Reradiation Patterns of Single Trees Based on Weighted Regression at $60 \mathrm{GHz}$}

\author{
Nuno R. Leonor, Rafael F. S. Caldeirinha, Telmo R. Fernandes, \\ and Manuel García Sánchez
}

\begin{abstract}
Due to their complex and inhomogeneous characteristics, the propagation and radiation parameters of trees and vegetation areas are very difficult and time consuming to obtain. This communication proposes a statistical method, using robust weighted local regression, to minimize the influence of the effect of the tree inhomogeneity on its reradiation pattern, allowing the evaluation of averaged reradiation functions from simple measurements. The proposed method was successfully applied to six tree specimens of both conifer and ficus species, at $60 \mathrm{GHz}$. Furthermore, once this empirical averaged function is obtained, first-order statistics can be applied to generate several simple reradiation functions, statistically identical to those obtained from measurements. Thus, allowing the full characterization of the tree under study.
\end{abstract}

Index Terms-Millimeter wave radio propagation, modeling, propagation measurements, scattering, vegetation.

\section{INTRODUCTION}

The presence of trees and vegetation areas in the radio path are very likely to severely affect the performance of radiowave communications, causing signal attenuation (absorption), scattering, and/or depolarization [1], [2].

Despite a plethora of propagation models available in the literature, one of the main issues while using or developing a vegetation model is the extraction of their input parameters. Empirical and semiempirical models [3]-[5] often use simple exponential equations with a very narrow set of input parameters. Notwithstanding, the accuracy of each one of these models is limited for scenarios outside the model scope [6]. Theoretical models that make use of radiowave theory to evaluate

Manuscript received March 13, 2015; revised July 01, 2015; accepted August 24, 2015. Date of publication August 27, 2015; date of current version October 28, 2015. This work was supported in part by the Portuguese Government, Portuguese Foundation for Science and Technology, FCT, through the financial support provided under the POPH/FSE funding, in part by the Instituto de Telecomunicações, Portugal, and in part by the Spanish Ministry of Economy and Competitiveness, Project no. TEC2014-55735C03-3R.

N. R. Leonor is with the Instituto de Telecomunicações, Leiria 2411-901, Portugal, and also with the Departmet of Teoria do Sinal e Communicacións, University of Vigo, Vigo 36200, Spain.

R. F. S. Caldeirinha and T. R. Fernandes are with the Instituto de Telecomunicações, Leiria 2411-901, Portugal, also with the School of Technology and Management, Polytechnic Institute of Leiria, Leiria 2411-901, Portugal, and also with the School of Engineering, University of South Wales, Treforest CF37 1DL, U.K. (e-mail: rafael.caldeirinha@ ipleiria.pt).

M. G. Sánchez is with the Department of Teoria do Sinal e Communicacins, Universidade de Vigo, Vigo 36200, Spain.

Digital Object Identifier 10.1109/TAP.2015.2474126 the total scattered field [7]-[10] are able to provide much more reliable signal predictions. However, the required detailed physical description of the trees present in the simulation path, e.g., leaf density, occupied canopy area, thickness and orientation [11], may become cumbersome for larger vegetation areas.

On the other hand, radiative energy transfer (RET)-based models [12]-[14] and a similar approach based on ray-tracing algorithms presented in [15], allow, to a certain degree, the detailed description of the simulation scenario. This includes the tree distribution and make use of the prior knowledge of the reradiation function of the trees under simulation, in order to define a set of input parameters required for the model engine.

Nonetheless, due to the characteristic inhomogeneity of trees, their reradiation function is dependent on the particular orientation of the tree around its vertical axis with respect to the transmitter. Thus, in order to obtain reliable reradiation measurement results, the scattered field around the tree should be recorded for several incidence angles to mitigate the tree orientation dependency, which in turns, can be rather prohibitive. For that purpose, an empirical method to minimize the influence of the specific tree specimen used for the reradiation measurements, thus simplifying the input parameter extraction of various propagation model present in the literature, is proposed.

This communication is then organized as follows. In Section II, a thorough characterization of the signal reradiation phenomena of several isolated tree specimens is presented. Section III addresses the development of a method to obtain the averaged reradiation function of a single tree from simple reradiation measurements. For that purpose, a parametric analysis on the input parameters of the robust locally weighted regression method is performed. The performance of proposed method is assessed against reradiation measurements of three conifer and three Ficus benjamina trees, performed in a controlled environment at $60 \mathrm{GHz}$. In Section IV, a practical application of the proposed method is presented. Finally, conclusion and directions for further work are drawn in Section V.

\section{Reradiation Functions of Single TREeS}

\section{A. Measurement Geometry and Procedure}

Measurements intending to record the reradiation pattern of single trees, at various incidence angles, were conducted in a controlled environment. For that purpose, the geometry depicted in Fig. 1 was assembled in an anechoic chamber, where the transmitter was set at three different positions. At each receiver position, the incidence point on the tree under measurement (TUM) was kept constant, thus implying three different incidence $\theta$ angles. The receiver was set to be at $1.4 \mathrm{~m}$ from ground level, and it was rotated around the tree at a constant distance of $1.3 \mathrm{~m}$, within an angular range from $\phi=-120^{\circ}$ to $\phi=120^{\circ}$. Additionally, for each step of the receiver arm rotation, the TUM was rotated around its vertical axis $360^{\circ}$ in an increment of $1^{\circ}$. The received signal level was recorded continuously during the tree rotation. For each tree rotation, 140000 samples were recorded. This allows one to record several samples of the reradiation pattern of the TUM.

\section{B. Measurement System Overview}

The measurements carried out throughout this communication were performed at $60.6 \mathrm{GHz}$. For that purpose, a transmitter comprising a signal generator $(\mathrm{SG})$, to provide a continuous-wave $(\mathrm{CW})$ tone at $600 \mathrm{MHz}$, was used. Additionally, a 15-GHz phase-locked loop (PLL) 